shocked when diagnosed and anxious about what would happen and wondered why they had such a disease, (5) Choice of treatment; patients were afraid of the side effects. They wanted to make a decision discussing with their doctor, but they could not understand the explanation about drugs well and, therefore, followed the doctor's opinion. (6) Change of treatment; a biological agent was often recommended. Patients were also worried about side effects and the financial burden. (7) Remission or stable phase; they felt better mentally too, however, they often felt anxiety about disease flare, side effect of drugs and financial burden, and (8) Flare and remission; patients felt shocked and disappointed when RA flared, and then, they noticed that patients with RA had alternating periods of relapse and remission and they had to live with RA.

These interviews revealed repeated worsening and improvement of symptoms and many similar repeated psychological reactions such as anxiety, shock, denial, conflict, acceptance, giving up and relief. To cope with these fluctuating disease and mental conditions, patients were supported by educational and psychological assistance, timely consultations, social life help from nurses and support from their family. The patients considered a trusting relationship with their doctors is necessary. The patients had also realized through their experience the importance of enhancing their own abilities, such as decision-making, prevention of infections and self-management skills. Moreover, they noticed that it is important to have their own goals including hobbies and work.

Conclusion: This study elucidated the common behaviors of patients with RA, the impact of RA on their psychological state and daily and social life, and the required support. The psychological condition and daily and social life also had a great influence on medical behavior. Therefore, psychosocial support and establishment of trust between healthcare professionals and patients are crucial. In addition, improving patients' self-management skills including self-efficacy and empowerment is also necessary. As patients with RA often feel anxious in various situations and expect nurses' support, nurses should listen to patients, pay attention to their concerns and anxieties, and show a solution-oriented attitude. In order for patients to feel at ease in their Patient Journey, nurses should sail with them while maintaining a patient-centered perspective.

Disclosure of Interests: None declared

DOI: 10.1136/annrheumdis-2021-eular.3029

\section{AB0895-HPR A MODERATED MEDIATION ANALYSIS FOR POOR ORAL HEALTH-RELATED QUALITY OF LIFE IN PATIENTS WITH BEHÇET'S DISEASE}

E. N. Çakır ${ }^{1}$, Y. Yenisoy ${ }^{1}$, A. Kapusuz ${ }^{1}$, K. Abacar ${ }^{2}$, F. Alibaz-Oner ${ }^{2}$, M. Yay $^{3}$, Ü. Karaçaylı ${ }^{4}$, N. Inanc ${ }^{2}$, T. Ergun ${ }^{5}, \underline{H}$. Direskeneli ${ }^{2}$, G. Mumcu ${ }^{6} .{ }^{1}$ Marmara University, Institute of Health Sciences, Istanbul, Turkey; ${ }^{2}$ Marmara University, Division of Rheumatology, Istanbul, Turkey; ${ }^{3}$ Mimar Sinan Fine Arts University, Department of Statistics, Istanbul, Turkey; ${ }^{4}$ Health Sciences University, Department of Oral and Dental Surgery, Ankara, Turkey; ${ }^{5}$ Marmara University, Department of Dermatology, Istanbul, Turkey; ${ }^{6}$ Marmara University, Department of Health Management, Istanbul, Turkey

Background: Since oral ulcer is a common clinical manifestation, oral health-related quality of life (OHRQoL) is affected by the presence of oral ulcer poorly in patients with Behcet's disease (BD).

Objectives: The aim of the study was to examine associated factors for poor OHRQoL status to improve treatment outcomes in patients with $\mathrm{BD}$.

Methods: In this retrospective study, 339 BD patients (F/M: 179/160, mean age: $36,13 \pm 9,81$ years) included. Data were collected by a structured questionnaire regarding treatment expectation, Oral Health Impact Profile-14 (OHIP-14) questionnaire for OHRQoL, oral ulcer activity, and disease severity score. Treatment expectation was coded by a 5-point Likert type scale (1: symptoms were completely cured vs 5: symptoms were worsened). OHIP-14 score was between " 0 point (the best score)" and "56 points (the worst score)." The disease severity score was calculated according to the organ involvement. Then, patients were categorised as mucocutaneous involvement $(n=181)$ and severe organ involvement $(n=158)$. After preliminary analysis, a Moderated Mediation analysis was carried out.

Results: Oral ulcer activity was seen in $63,4 \%$ of the group $(n=215)$. OHIP-14 score $(22,05 \pm 16,47)$ was correlated with Treatment expectations $(2,35 \pm 0,98)$ ( $r: 0,36 \mathrm{p}=0.000$ ) in the group. Poor scores of OHIP-14 and Treatment expectations were found in patients with active oral ulcers (mucocutaneous involvement: $27,56 \pm 14,55 ; 2,68 \pm 0,87$, vs severe organ involvement: $28,51 \pm 17,25 ; 2,43 \pm 0,99$ ) compared to those of inactive patients (mucocutaneous involvement: $13,38 \pm 12,23$; $1,81 \pm 0,73$ vs severe organ involvement: $8,79 \pm 10,19 ; 1,85 \pm 1,02)(p=0.000$ for all). Patients with active oral ulcers were younger $(35,09 \pm 9,52)$ than the others $(39,57 \pm 10,06)$ in the mucocutaneous involvement group $(p=0.0011)$ whereas a similar relation was not seen in the severe organ involvement $(p=0.233)$.

In the moderated mediation analysis, it was found that OHIP-14 score was increased by the presence of oral ulcer in the direct path $(p=0.0000)$ and the negative Expectation of the treatment as a first mediator $(p=0.0001)$ in the indirect path. Oral ulcer activity was associated with younger patients $(p=0.0039)$. This effect was seen especially in patients with mucocutaneous involvement that had a moderator effect $(p=0.0040)$. In addition, age was found to be a second mediator for the poor OHIP-14 score $(p=0.0053)$. Mediators and the moderator were found to effective for OHIP-14 score a bootstrap analysis with 5000 replications. Conclusion: OHRQoL was affected by oral ulcer activity and poor treatment expectations. Age was also found to be another critical factor for OHRQoL.

Disclosure of Interests: None declared

DOI: 10.1136/annrheumdis-2021-eular.3043

\section{AB0896-HPR THE EVALUATION OF TYPE D PERSONALITY AND SELF-ESTEEM IN PATIENTS WITH FIBROMYALGIA}

N. Gokcen ${ }^{1}$, I. Coskun Benlidayi ${ }^{2}$, L. Tamam ${ }^{3}$, M. E. Demirkol ${ }^{3}$, C. Yesiloglu ${ }^{3}$, R. Guzel ${ }^{2}{ }^{1}$ Cukurova University Faculty of Medicine, Department of Physical Medicine and Rehabilitation, Division of Rheumatology, Adana, Turkey; ${ }^{2}$ Cukurova University Faculty of Medicine, Department of Physical Medicine and Rehabilitation, Adana, Turkey; ${ }^{3}$ Cukurova University Faculty of Medicine, Department of Psychiatry, Adana, Turkey

Background: Fibromyalgia (FM) is a condition characterized by chronic widespread pain, fatigue, sleep disturbances. Etiopathogenesis remains unclear; however, growing data regarding the effect of psychosocial factors on disease etiopathogenesis have drawn attention. Psychosocial elements such as type D personality and low level of self-esteem might increase disease burden (1). Accordingly, many researchers so far, have examined psychological parameters in FM and a limited number of studies focused on the personality type among patients with FM (2-5). Objectives: The current study aimed to investigate type $D$ personality and self-esteem in patients with FM.

Methods: Female patients with fibromyalgia and age-matched healthy controls were enrolled in this cross-sectional study. Type D personality was evaluated by using the type D scale (DS-14). The Rosenberg self-esteem scale (RSES) was used to assess self-esteem and psychosomatic symptoms. Psychological status was evaluated by the Beck Depression and Beck Anxiety Inventories. Fibromyalgia Impact Questionnaire (FIQ) was performed to assess functional impairment and health status in patients with FM. The potential confounders of type D personality were evaluated by binary logistic regression analysis.

Results: Seventy patients with FM and 60 controls were included. Type D personality was observed in $58.6 \%$ of the patients and in $21.7 \%$ of the control group $(p<0.001)$. When compared to patients with non-type $D$ personality, those with type $D$ personality revealed lower self-esteem and poorer health status $(p<0.05$ for both), higher anxiety and depression scores ( $p<0.001$ for both). According to binary logistic regression analysis, depression $(\beta=1.178, \mathrm{Cl} 95 \% 1.055-1.316$ $p=0.004)$ and lower education level $(\beta=0.818, C l 95 \% 0.682-0.982, p=0.031)$ were significantly related to type D personality in FM (Table 1).

Conclusion: Type D personality is more common in female patients with FM when compared to healthy women. Patients with type D personality have lower level of self-esteem when compared to those with non-type D personality. Depression and lower education level are potential confounders of type D personality in FM.

\section{REFERENCES:}

[1] Bazzichi L, Giacomelli C, Consensi A, Giorgi V, Batticciotto A, Di Franco M, Sarzi-Puttini P (2020) One year in review 2020: fibromyalgia. Clin Exp Rheumatol 38 Suppl 123(1):3-8.

[2] Conversano C, Marchi L, Ciacchini R, Carmassi C, Contena B, Bazzichi LM, Gemignani A (2018) Personality Traits in Fibromyalgia (FM): Does FM Personality Exists? A Systematic Review. Clin Pract Epidemiol Ment Health 14:263.

[3] Türkoğlu G, Selvi Y (2020) The relationship between chronotype, sleep disturbance, severity of fibromyalgia, and quality of life in patients with fibromyalgia. Chronobiol Int 37(1):68-81.

[4] Garip Y, Güler T, Bozkurt Tuncer Ö, Önen S (2019) Type D Personality is Associated With Disease Severity and Poor Quality of Life in Turkish Patients With Fibromyalgia Syndrome: A Cross-Sectional Study. Arch Rheumatol 35(1):13-19.

[5] Ablin JN, Zohar AH, Zaraya-Blum R, Buskila D (2016) Distinctive personality profiles of fibromyalgia and chronic fatigue syndrome patients. PeerJ 4:e2421.

Table 1. Binary logistic regression analysis for the evaluation of potential confounders of type $D$ personality in patients with fibromyalgia.

\begin{tabular}{lllll}
\hline & Wald & SE & $\beta(95 \% \mathrm{Cl})$ & $\mathbf{p}$ \\
\hline Education (years) & 4.664 & 0.093 & $0.818(0.682-0.982)$ & 0.031 \\
Occupation duration (years) & 0.116 & 0.037 & $1.013(0.942-1.089)$ & 0.733 \\
Self-esteem scale (D1) & 0.369 & 0.423 & $0.774(0.338-1.771)$ & 0.544 \\
BDI & 8.446 & 0.056 & $1.178(1.055-1.316)$ & 0.004 \\
BAI & 0.789 & 0.032 & $1.029(0.966-1.095)$ & 0.374
\end{tabular}

BDI: Beck Depression Inventory, BAI: Beck Anxiety Inventory, SE: standard error, Cl: confidence interval 